\section{PRDI-BF1 and PRDI-BF1 $\beta$ isoform expressions correlate with disease status in multiple myeloma patients}

\author{
Gabriele Buda, Francesca Guerrini, \\ Sara Galimberti, Enrico Orciuolo, \\ Simone Pacini, Elisa Mazzantini, \\ Mario Petrini \\ Department of Clinical and \\ Experimental Medicine, U.O. \\ Hematology, University of Pisa, Italy
}

\begin{abstract}
Human positive regulatory domain binding factor 1 (PRDI-BF1 or BLIMP-1) is a transcription factor that acts as a master regulator and has crucial roles in the control of differentiation and in maintaining survival of plasma cells (PC). The PRDMI gene, which codifies for PRDI-BF1, contains an alternative promoter capable of generating a PRDI-BF1 deleted protein (called PRDI-BF1 $\beta$ ), which lacks 101 amino acids comprising most of the regulatory domain. PRDI-BF $1 \beta$ has been detected in relevant quantities especially in multiple myeloma cell lines (U266 and NCI- H929). The first aim of the study was to compare, using real time polymerase chain reaction (RT-PCR), the levels of PRDI-BF1 and PRDI-BF1 $\beta$ in myeloma patients and in normal human bone marrow. The second step was the examination of the expression of PRDI-BF1 and PRDI-BF1 $\beta$ isoform depending on disease status and treatment response. We demonstrate the correlation of PRDI-BF1 and the shorter PRDI-BF1 $\beta$ isoform protein levels with the clinical evolution and the management of myeloma patients.
\end{abstract}

\section{Introduction}

Human positive regulatory domain I binding factor 1 (PRDI-BF1 or BLIMP-1) is a transcription factor that acts as a master regulator and has crucial roles in the control of differentiation and in maintaining survival of plasma cells (PC). ${ }^{1}$ PRDI-BF1 essentially functions as a repressor, causing exit from cell cycling and the extinction of the expression of several genes critical for B-cell development at earlier stages. ${ }^{2,3}$ The PRDM1 gene, which codifies for PRDI$\mathrm{BF} 1$, contains an alternative promoter capable of generating a PRDI-BF1 deleted protein (called PRDI-BF1 $\beta$ ), which lacks 101 amino acids comprising most of the regulatory domain. ${ }^{3}$ PRDI-BF $1 \beta$ has been detected in relevant quantities especially in multiple myeloma (MM) cell lines (U266 and NCI- H929). Since this molecule contains the DNA-binding domain but bears a disrupted regulatory domain, its function remains to be elucidated. ${ }^{4}$

Moreover, Blimp-1/PRDM1 binding is contained in the promoter region of CS1, (CRACC/CD319/SLAMF7) a member of SLAM (Signaling Lymphocyte Activation Molecule) that is upregulated in multiple myeloma cells and contributes to clonogenic growth and tumorigenicity. ${ }^{2-4}$ Mutating the Blimp-1/PRDM1 site at -750 to -746 decreased the transcriptional activity of CS1 promoter implicating a trans-activating function of Blimp-1/PRDM1 in human CS1 gene regulation. ${ }^{4-6}$ The potential role of Blimp-1/PRDM1 in multiple myeloma, may help in understanding the progression of the disease and the potential specific function of new drugs as elotuzum$\mathrm{ab}$, that selectively blocks CS1.

Recent data also showed that treatment with lenalidomide caused ubiquitination and proteasomal degradation of PRDI-BF1, leading to the de-repression of a new PRDIBF1 direct target, CULLIN 4A (CUL4A). Accordingly, lenalidomide-induced cell death was partially rescued by reintroduction of PRDI-BF1.5,7 Anyway, no data are still available about the different expressions of PRDI-BF1 and its isoform beta during the clinical evolution and the management of Myeloma patients.

\section{Materials and Methods}

In our department, we selected 15 multiple myeloma patients and 5 healthy subjects. Eight Patients have been previously treated with VTD (bortezomib, thalidomide, dexametason $\times 4$ cycles) induction regimen and autologous bone marrow transplant (with melphalan 200/sqm as conditioning), obtained a VGPR (very good partial response) or better response and were in periodical and clinical follow up. Three patients, reached only a partial response after induction therapy and the remaining four patients were selected in relapsed or progressed disease after the treatment. All patient, with inform written consent and in accordance to the declaration of Helsinki, performed a bone marrow analysis and aspirate for assessment of blood samples in two different points. Bone marrow samples in the eight patients in VGPR status, were taken at 6 months of distance during the follow up. The three patients at diagnosis, col-
Correspondence: Gabriele Buda, Department of Clinical and Experimental Medicine, U.O. Hematology, University of Pisa, Via Roma 67, 56100, Pisa, Italy.

E-mail: ga.buda@libero.it

Key words: Hematology, Multiple Myeloma, multi drug resistance.

Contributions: the authors contributed equally.

Conflict of interest: the authors declare no potential conflict of interest.

Received for publication: 23 April 2017.

Revision received: 9 October 2017.

Accepted for publication: 9 October 2017

This work is licensed under a Creative Commons Attribution-NonCommercial 4.0 International License (CC BY-NC 4.0).

CCopyright G. Buda et al., 2017

Licensee PAGEPress, Italy

Hematology Reports 2017; 9:7201

doi:10.4081/hr.2017.7201

lected bone marrow samples at the baseline and one month after the induction therapy. The last four patients were analyzed at the time of progression and one month after the salvage treatment.

The first aim of the study was to compare, using real time polymerase chain reaction (RT-PCR), the levels of PRDI-BF1 and PRDI-BF1 $\beta$ in myeloma patients $(n=15)$ and normal human bone marrow $(\mathrm{n}=5)$.

The second step was the examination of the expression of PRDI-BF and PRDI-BF1 $\beta$ isoform depending on disease status and treatment response. In order to perform a relative quantitative RT-PCR analysis of both factors, two different oligonucleotides pairs were used, one detecting both the $\alpha$ and $\beta$-isoforms, and the other recognizing only the PRDI-BF1 $\beta$ isoform.

Mononuclear cells were isolated by fractionation on Lymphosep (Lymphocyte Separation Media; Carlo Erba Reagents, Milan, Italy) gradient.

Total RNA was extracted using UPzol RNA Isolation (Biotechrabbit, Berlin, Germany) and reverted using RevertAid First Strand cDNA Synthesis Kit (Thermo Scientific, Waltham, MA USA), according to the manufacturer's protocols.

Primer mixes and TaqMan probes were purchased from Bio-Rad (Hercules, CA USA); assay references: qHsaCIP0039044 for PRDI-BF1 $(\alpha$ and $\beta$, amplifying a fragment of $115 \mathrm{pb}$ between exon-4 and exon-5 -common to both isoforms-) and dHsaCPE5031597 for GAPDH (used as internal reference). 
For PRDI-BF1 $\beta$ specific detection, primer and TaqMan probe were synthesized using the Assays-by-Design Service (BioRad, Hercules, CA USA) (Forward: CTCTGTGGTGGGTAATC, Reverse: CCCTCTGGAatAGATCTTTTC, Probe: 5, 6-FAM-TTTGAGGCAGCTCCTTAAATGAACATT- 3' Iowa Black ${ }^{\circledR}$ FQ (amplifying a fragment of $126 \mathrm{pb}$ ). ${ }^{8}$

QT-PCR reactions were run in duplicate using an iCycler and iQ software Version 2.1 (Bio-Rad, Milan, Italy) and duplicates were averaged. Threshold cycles $(\mathrm{Ct})$ for the genes of interest were normalized to the $\mathrm{Ct}$ value of the control gene (GAPDH); the relative quantitative values were calculated using the relative quantification according to the delta-CT method (Pfaffl method). ${ }^{9}$

\section{Results}

PRDI-BF1 was expressed at considerable levels in all samples tested and PRDIBF1 $\beta$ was well expressed too. In all MM patients the mean expression of PRDI-BF1 was 5.92 (range 1.00-21.75) fold higher than in healthy controls and the mean expression of beta isoform was 8.02 fold higher than in healthy subjects (range 1.0029.00). When we examined the expression of PRDI-BF and PRDI-BF1 $\beta$ isoform, depending on disease status and treatment response, we showed that in 8 of 15 patients already in follow up for at least a VGPR or better status, the levels of PRDI-BF and PRDI- BF1 $\beta$ isoform were quite stable in each patient at the two different points at 6 months of distance. These values were only a little bit higher when compared to healthy subjects, respectively a mean level of 2.923.01 for PRDI-BF in the two different points of follow up and mean level 3.3-4.8 for PRDI-BF1 $\beta$ isoform.

In 3 patients who reached only a partial response of disease after the induction regimen, the levels of PRDI-BF reduced from a mean of 10.83 (range 7.72-14-46) fold higher than in health subjects to a mean of 5.05 after the end of induction therapy (range 2.67-8.9). In relapsed/progressed subgroup of patients (4/15), the high levels of PRDI-BF confirmed a relation to the disease status in particular with beta isoform that showed a mean of 11.6 fold higher of transcript level (range 9.1-12.89) compared to healthy subjects (Figure 1 and Table 1).

\section{Discussion and Conclusions}

Although much is known about genes involved in the differentiation of $\mathrm{B}$ cells to plasma cells, there is less information about genes required for maintenance of plasma cell in the bone marrow. ${ }^{10,11}$ The physiological significance of the differential expression of the two PRDI-BF1 isoforms remains to be elucidated, as described in other PRDM family members, expressed in two different isoforms. ${ }^{3,6}$ In addition, the tumorigenic capacity of a defective PRDIBF1 has been already observed in human diffuse large B-cell lymphoma. Therefore, it is conceivable that the overexpression of PRDI-BF1 $\beta$ isosform more specifically detected in MM patients samples could con-

Table 1. Mean levels of PRDI-BF and PRDI-BF beta isoform in patients compared to healthy subjects in the two different controls (during the follow up for good responders patients and before and after treatment for the other two groups).

\begin{tabular}{lcccc} 
& \multicolumn{2}{c}{ PRDI-BF } & \multicolumn{2}{c}{ PRDI-BF beta } \\
& Mean & Mean & Mean & Mean \\
Good responders patients & 2.92 & 3.01 & 3.81 & 4.30 \\
Partial responders patients & 10.83 & 5.05 & 8.81 & 6.21 \\
\hline Not responders patients & 5.9 & 7.25 & 6.1 & 17.25 \\
\hline
\end{tabular}
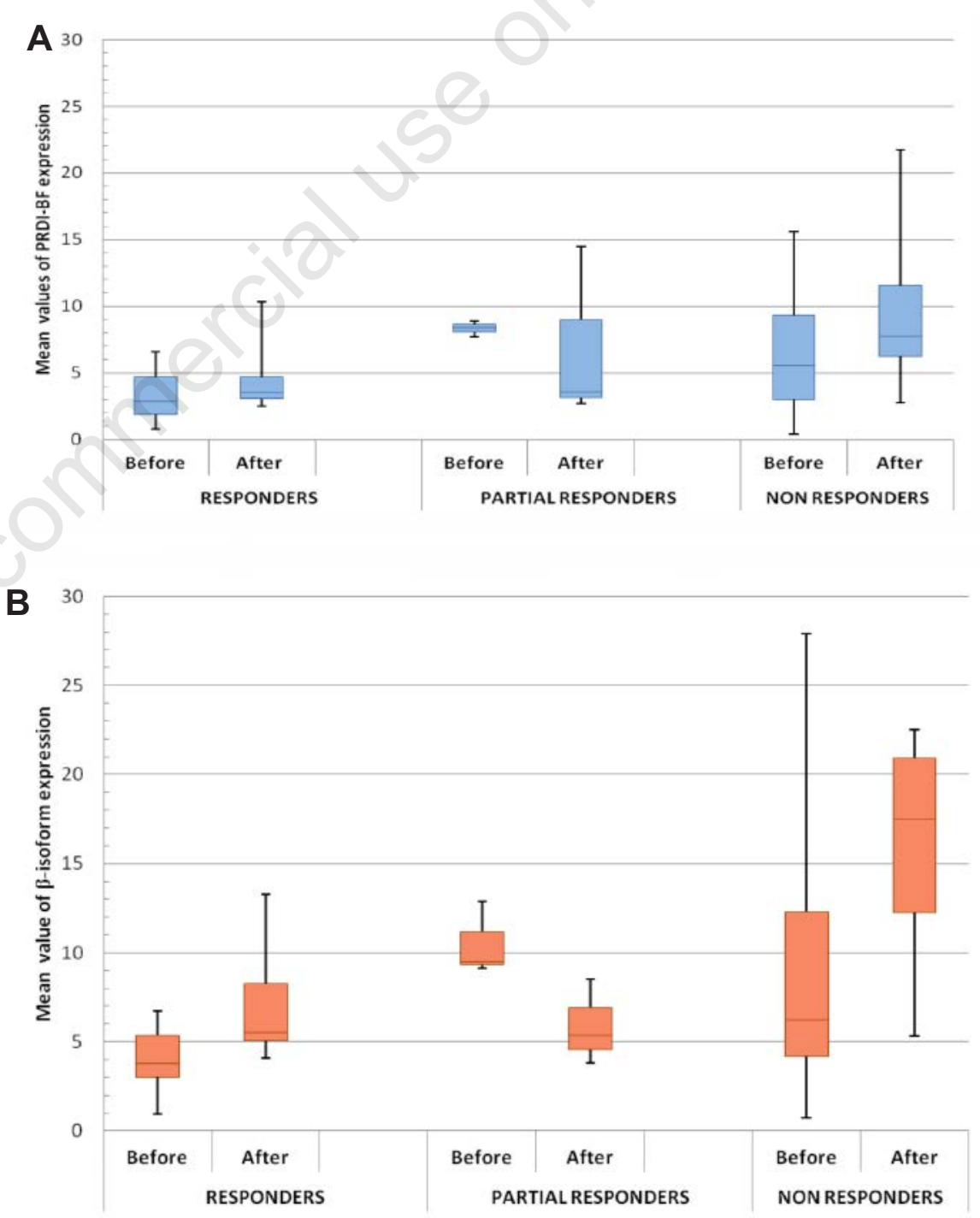

Figure 1. Expression of PRDI-BF (A) and beta isoform (B) in MM patients related to disease status. 
tribute to progression into the tumoral state, in particular determining the loss of polyclonality accompanied by proliferation of a monoclonal cell type. ${ }^{12,13}$

Our data suggest that the total level of PRDI-BF and in particular the level of PRDI-BF1 $\beta$ are clearly detectable in MM patients. The expression of these genes could be a feature distinguishing between remission of active disease of MM. Importantly, at the best of our knowledge, this is the first report where the PRDI-BF1 and the shorter PRDI-BF1 $\beta$ protein have been correlated with the disease status. Previous studies had also speculated if PRDI-BF1 over expression can be crucial for the control of plasma cell differentiation and in maintaining survival of plasma cell. ${ }^{4}$ In particular PRDI-BF1 $\beta$ isoform over expression seemed to be more specific for maintaining clonality of malignant plasma cells. ${ }^{3,5}$

We demonstrate the correlation of PRDI-BF1 and the shorter PRDI-BF1 $\beta$ isoform protein levels with the disease status of Myeloma patients. In particular patients with stable myeloma in at least VGPR, showed only low levels of PRDI-BF1 and PRDI-BF1 $\beta$ if compared to the healthy subjects. The levels of PRDI-BF1 and in particular of its beta isoform are directly correlated with the disease status and the different response to the treatment. Patients with initial response to the therapy showed a reduction of transcription levels that on the contrary were marked up regulated in refractory patients. Differences in transcriptions levels of PRDI-BF1 and the PRDI-BF1 $\beta$ really reflected the clinical status of the patients and were well measurable after the first month at the end of the therapy. These
Results confirm a central role of PRDI-BF1 that could be linked to the mechanism of function of some new drugs use in MM treatment. in particular, PRDI-BF1 is required for XBP-1 induction, a transcriptional activator necessary for plasma cell survival because reduction levels of activated XBP1 determined by proteasome inhibitors causes rapidly cell apoptosis. ${ }^{7}$ PRDI-BF1 and the PRDI-BF1 $\beta$ can be considered a crucial point for understanding efficacy and limits of many new drugs used in MM.

\section{References}

1. Shapiro-Shelef M, Lin KI, Savitsky D, et al. Blimp-1 is required for maintenance of long-lived plasma cells in the bone marrow. J Exp Med 2005;202:1471-6.

2. Ocaña E, González-García I, Gutiérrez $\mathrm{NC}$, et al. The expression of PRDI-BF1 beta isoform in multiple myeloma plasma cells. Haematologica 2006;91:157980.

3. Kim JR, Mathew SO, Mathew PA. Blimp-1/PRDM1 regulates the transcription of human CS1 (SLAMF7) gene in NK and B cells. Immunobiology 2016;221:31-9.

4. Klein B, Tarte K, Jourdan M, et al. Survival and proliferation factors of normal and malignant plasma cells. Int J Hematol 2003;78:106-13.

5. Lin FR, Huang SY, Hung KH, et al. ASK1 promotes apoptosis of normal and malignant plasma cells. Blood 2012;120:1039-47.

6. Györy I, Fejér G, Ghosh N, et al.
Identification of a functionally impaired positive regulatory domain I binding factor 1 transcription repressor in myeloma cell lines. J Immunol 2003;170: 3125-33.

7. Hung KH, Su ST, Chen CY, et al. Aiolos collaborates with Blimp-1 to regulate the survival of multiple myeloma cells. Cell Death Differ 2016;23:1175-8.

8. Livak KJ, Schmittgen TD. Analysis of relative gene expression data using realtime quantitative PCR and the 2(-Delta Delta C(T)) Method. Methods 2001;25:402-8.

9. Pfaffl MW. Relative quantification. In: Dorak T, ed. Real time PCR, vol 2. La Jolla: International University Line; 2006. pp 63-82.

10. Borson ND, Lacy MQ, Wettstein PJ. Altered mRNA expression of Pax 5 and Blimp-1 in B cells in multiple myeloma. Blood 2002;100:4629-39.

11. Garcia JF, Roncador G, García JF, et al. BLIMP-1 expression in multiple B and T-cell lymphoma. Haematologica 2006;91:467-74.

12. Xia Y, Xu-Monette ZY, Tzankov A, et al. Loss of PRDM1/BLIMP-1 function contributes to poor prognosis of activated B-cell-like diffuse large B-cell lymphoma. Leukemia 2016. [In Press].

13. Nagy M, Chapuis B, Matthes T. Expression of transcription factors Pu.1, Spi-B, Blimp-1, BSAP and oct-2 in normal human plasma cells and in multiple myeloma cells. $\mathrm{Br} \mathrm{J}$ Haematol 2002;116:429-35. 